\title{
Frequency stabilization of ambience-isolated internal-mirror He-Ne lasers by thermoelectric-cooling thermal compensation
}

\author{
Hamidreza Shirvani-Mahdavi $^{1} \cdot$ Yaghoubi Narges $^{1}$
}

Received: 21 April 2016/ Accepted: 8 August 2016/Published online: 16 August 2016

(c) The Author(s) 2016. This article is published with open access at Springerlink.com

\begin{abstract}
An approach for frequency stabilization of an ambience-isolated internal-mirror $\mathrm{He}-\mathrm{Ne}$ laser $(632.8 \mathrm{~nm})$ utilizing temperature control of the laser tube with Peltier thermoelectric coolers is demonstrated. Measurements indicate that there are an optimal temperature $\left(23^{\circ} \mathrm{C}\right)$ and an optimal discharge current $(5.5 \mathrm{~mA})$ of laser tube for which the laser light power is separately maximized. To prevent the effect of fluctuation of discharge current on the laser stability, an adjustable current source is designed and fabricated so that the current is set to be optimal $(5.50 \pm 0.01 \mathrm{~mA})$. To isolate the laser tube from the environment, the laser metallic box connected to two Peltier thermoelectric coolers is surrounded by two thermal and acoustic insulator shells. The laser has two longitudinal modes very often. Any change in the frequency of longitudinal modes at the optimal temperature is monitored by sampling the difference of longitudinal modes' intensities. Therefore, using a feedback mechanism, the current of thermoelectric coolers is so controlled that the frequency of modes stays constant on the gain profile of the laser. The frequency stability is measured equal to $1.17 \times 10^{-9}$ $(\sim 2700 \times)$ for less than $1 \mathrm{~min}$ and $2.57 \times 10^{-9}$ $(\sim 1200 \times)$ for more than $1 \mathrm{~h}$.
\end{abstract}

Hamidreza Shirvani-Mahdavi

hsm@iauctb.ac.ir

Yaghoubi Narges

n.yaghoubi.ph@gmail.com

1 Photonics Research Laboratory, Department of Physics, Islamic Azad University (Central Tehran Branch), Tehran, Iran
Keywords Frequency stabilization - Optimal temperature Peltier thermoelectric cooler $\cdot$ Ambience-isolated $\mathrm{He}-\mathrm{Ne}$ laser

\section{Introduction}

The He-Ne laser is commonly used in laboratories as a simple and convenient light source in various experiments and measurements. The coherence time of the laser is very important for many laser applications such as interferometry, speed measurements, laser gyroscopes, and so on. Among these applications, recently the generation of vector doughnut beams and purely single transverse mode vortex beams $[1,2]$ are also under consideration, for which the periodical change of the intensity and polarization distributions of the He-Ne lasers can significantly affect the final outcomes. Therefore, the thermally stabilized He-Ne lasers play a very important role to improve the experiments' results.

In recent years, many scientists, owing to a high ability of He-Ne lasers for stabilizing in the Doppler line width range, have focused their attention on the enhancement of coherence time. In 1972, Balhorn et al. established the frequency stabilization theory in this type of lasers for the first time [3]. To stabilize the frequency of laser light, they controlled the voltage of laser power supply by using intensity changes arising from temperature variations. Their effort leaded to the stability of $\Delta v / v=1 \times 10^{-7}$ $(30 \times)$. In addition, after 1973, Bannet et al. achieved the stability of $2 \times 10^{-8}(160 \times)$ by the heating coil method [4]. Since then, passive and active methods with different designs and techniques have been developed for frequency and amplitude stabilization of $\mathrm{He}-\mathrm{Ne}$ lasers, which has resulted in the stability of $\sim 10^{-16}\left(\sim 10^{10} \times\right)$ [5-18]. 
As seen in previous researches, in the first approximation, the stabilization is normally carried out by controlling the length of the laser. In the case of internal-mirror $\mathrm{He}-\mathrm{Ne}$ lasers, the length of the laser can be controlled by adjusting the temperature of the laser tube. For this purpose, generally, the current of a strip heater wound around the laser tube can be controlled by an electronic circuit. Although in this method one can achieve a quite high stability, $\sim 10^{-9}$ [19], using a heater may change the original features of laser tube, making the stabilization process more difficult due to additional temperature increase in the laser tube, which in turn reduces its lifetime. To overcome the problem, in 1982 Sasaki et al. [14] controlled the temperature of the laser tube by a cooling fan and obtained a stability of $\sim 10^{-8}$ by this procedure. In 2012, Qian et al. achieved a stability of $4.3 \times 10^{-9}$ by removing some drawbacks. They realized that the short-term stability may be limited by mechanical vibration of the fan and air flow, which can be improved by utilizing a mini cooling fan with lower vibration. In addition, to achieve a smoother rotation of the fan, they optimized the drive circuit [15]. Although the use of fan apparently prevents the temperature rise of the laser tube, the least disruption in the fan revolution adjustment severely affects short-term stability. To control the length of laser cavity, Ellis et al. utilized a Peltier thermoelectric cooler in 2010 and 2012 [16, 17]. They used a mixed mode within the optical cavity to stabilize the absolute laser frequency. But in the papers, the effect of laser plasma ambient temperature on the output power has not been examined.

In this paper, frequency stabilization of an internal-mirror $\mathrm{He}-\mathrm{Ne}$ laser is performed by the control of the laser tube's temperature using two thermoelectric coolers. First of all, the discharge current of the laser is adjusted at the operating point, and then, to prevent the effect of external factors, the laser is isolated from the environment via two thermal and acoustic insulator shells. The laser ambient temperature is controlled via an appropriate electronic circuit using two Peltier thermoelectric coolers around the temperature $23{ }^{\circ} \mathrm{C}$, so that the laser frequency can be stabilized. The advantage of this approach, in addition to adjusting the laser ambient temperature to maximize the laser light power, is to overcome the effect of ambient temperature variations on the laser performance. On the other hand, it should be noted that this method, in comparison to much other techniques, can be more cost-effective.

\section{Experimental}

In this study, an internal-mirror He-Ne laser (Model 2500, from supplier: Changsha Aube Didactic Equipment Co., Ltd.) oscillating at the wavelength $632.8 \mathrm{~nm}$, with the

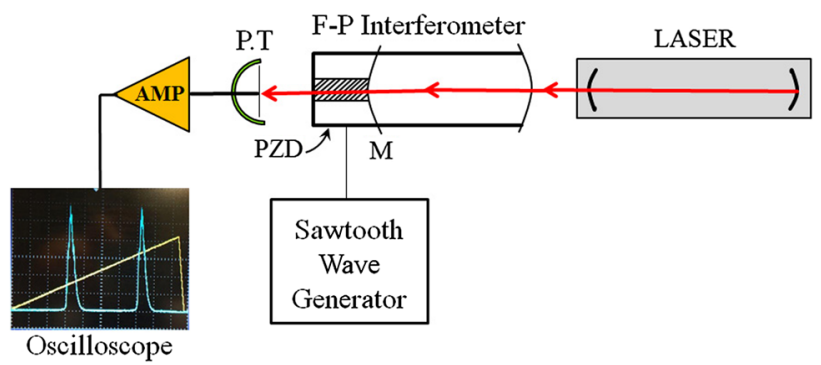

Fig. 1 A schematic setup for measuring laser modes by a scanning Fabry-Perot interferometer (SF-PI): $P Z D$ piezoelectric device, $M$ mirror of the SF-PI connected to PZD, P.T photodetector, AMP amplifier

maximum power $2.43 \mathrm{~mW}$ at the temperature $23{ }^{\circ} \mathrm{C}$, the cavity length $L=24.5 \mathrm{~cm}$, an axial mode spacing of about $c / 2 L=612 \mathrm{MHz}$, and without any Brewster windows, was used. The laser does not have any transverse modes. Initially, the number of laser longitudinal modes was measured by a scanning Fabry-Perot interferometer (SF-PI) (Fig. 1).

The longitudinal modes of laser were separated by changing the length of SF-PI by a piezoelectric device (PZD) connected to the mirror M. The sawtooth wave generator drives $\mathrm{PZD}$, which controls the vibration of mirror M. The laser light after transmitting through SF-PI is detected by the optical detector (PT), whose electronic signal after amplifying by the amplifier (AMP) is observed via the oscilloscope. The measurement shows that the laser has two longitudinal modes very often, but with the change of laser length arising from the thermal expansion; when one of modes is closed to the central frequency in the gain profile, the third mode may also appear.

\section{Determination of laser principal axes}

In gas lasers, owing to the induced anisotropy in the laser mirrors, there exists two orthogonal principal axes for the laser system at a plane perpendicular to the laser light propagation [20, 21]. The refractive indices and absorption coefficients in the direction of these two axes are normally different. On the other hand, calculations show that the polarization of each longitudinal mode in gas lasers is linear and in the direction of one of the principal axes. In addition, the polarizations of successive modes are perpendicular [22]. Here, the direction of laser principal axes was determined using an SF-PI. For this purpose, a polarizer was put in the front of the laser light whose polarization direction is generally arbitrary with respect to the principal axes $(x, y)$. Thus, the laser light after passing the polarizer consists of all longitudinal modes, except that the polarizer transmission axis coincides with each of the two principal axes $(x, y)$, which results in the elimination of 
one or two of the three modes, as shown in Fig. 2. In this method, the principal axes were determined with an accuracy equal to that of the polarizer calibration $\left( \pm 5^{\circ}\right)$.

\section{Laser stabilization system}

The laser stabilizing system was generally constructed from four parts: the power supply unit, the thermodynamic system, optical components and the stabilizing circuit (Fig. 3).

The power supply unit Due to the effect of voltage fluctuations of the city electricity and instability of the laser power supply on the ultimate limit of frequency stabilization, an appropriate adjustable current source was designed and fabricated to control the current of the laser discharge tube with the precision $\pm 0.01 \mathrm{~mA}$ (Fig. 4). $\mathrm{DZ}_{1}$ and $\mathrm{DZ}_{2}$ consisted of two $5.6 \mathrm{~V}$ Zener diodes, operating as a voltage reference with an approximately zero temperature coefficient. By altering the potentiometer $P_{1}$ and applying an adequate voltage to the inverting input of the operational amplifier $\left(\mathrm{OA}_{1}\right)$, the current of the laser tube can be

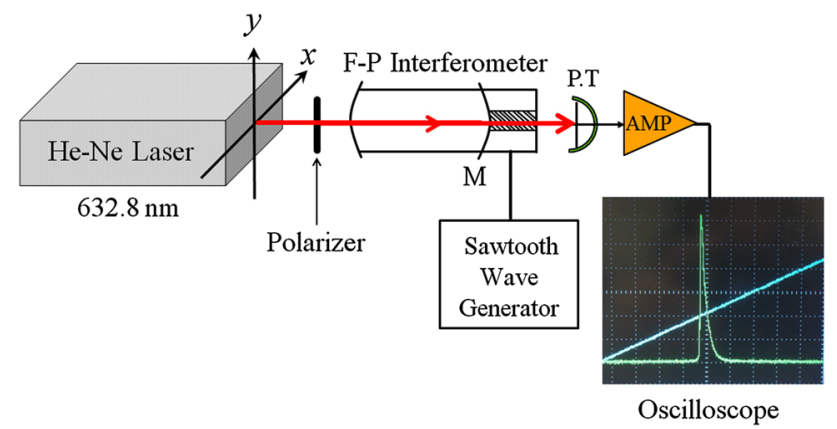

Fig. 2 Block diagram of laser principal axes measurement by SF-PI: $M$ mirror, $P . T$ photodetector, $A M P$ amplifier

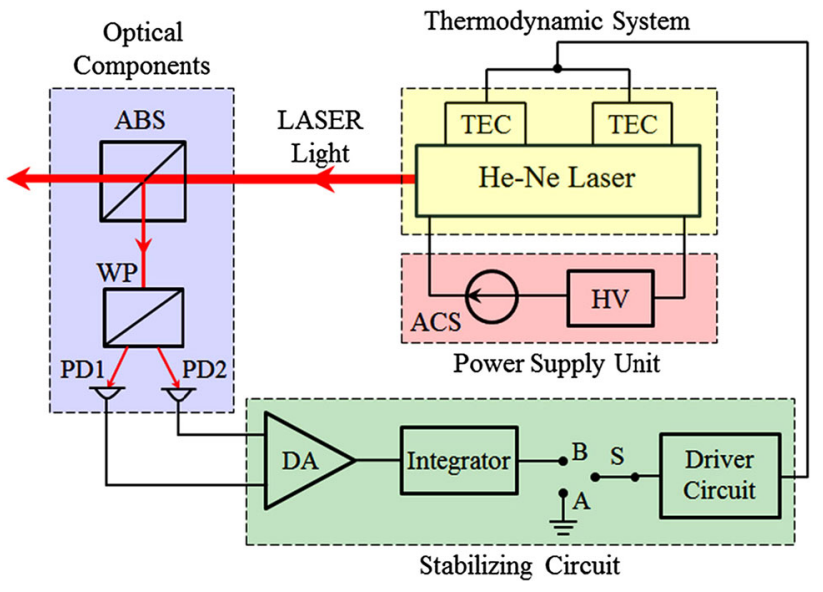

Fig. 3 The block diagram of the stabilization system: $H V$ highvoltage source, ACS adjustable current source, TEC thermoelectric cooler, $A B S$ asymmetrical beam splitter, $W P$ Wollaston prism, $P D 1$ and $P D 2$ photodetectors, $D A$ differential amplifier adjusted at an optimal value. Hereafter, any unwanted change in the laser current will be neutralized through the negative feedback loop, including the optocouplers $\mathrm{OP}_{1}$ and $\mathrm{OP}_{2}$, the resistor $R_{7}$ and the operational amplifier $\mathrm{OA}_{1}$. The Darlington transistors $\mathrm{DT}_{1}-\mathrm{DT}_{4}$, biased through the resistors $R_{1}-R_{4}$, provide a voltage ladder as a high-voltage transistor.

The thermodynamic system To avoid the effect of thermal and acoustic variations on the frequency stabilization of the laser, in addition to the metallic box of the laser an MDF box whose inner walls have completely been insulated by two thermal and acoustic insulation shells was used. Phenolic with chemical composition $\mathrm{C}_{6} \mathrm{H}_{5} \mathrm{OH}$ and thermal transfer coefficient of $0.018 \mathrm{~W} / \mathrm{m}{ }^{\circ} \mathrm{C}$ at $24{ }^{\circ} \mathrm{C}$ was utilized as the thermal insulator. On the other hand, the material consists of rubber grains of EPDM (ethylenepropylene diene terpolymer), which is made under the hot pressing process in the presence of anti-rupture materials and its acoustic insulation coefficient is $55 \mathrm{~dB}$, was exploited as the acoustic insulator. For keeping constant the temperature of the laser system, two thermoelectric coolers (with $70 \mathrm{~W}$ cooling capacity) were connected to the metallic box of the laser through an aluminum block with dimensions $(14 \times 7.5 \times 4) \mathrm{cm}$. To establish better connection, all surfaces were foremost polished very well. Furthermore, to achieve the best heat energy conduction from the laser metallic box to the aluminum block and finally to the Peltier coolers, the paste of zinc oxide $\left(\mathrm{ZnO}_{2}\right)$ was utilized as the interface. The reason for the use of $\mathrm{ZnO}_{2}$ is its much larger thermal conductivity $(200 \times)$ compared to air existing in the microscopic bumps between surfaces. To connect the Peltier thermoelectric coolers to the aluminum block and also the aluminum block to the laser metallic box, eight springs were used. The hot surface of each thermoelectric cooler was connected to an aluminum heat sink with dimensions of $(7.6 \times 6.7 \times 4) \mathrm{cm}$ that is cooled by a high-speed fan (Fig. 5).

Optical components As seen from Fig. 3, a slight part of the laser light is sampled by the asymmetrical beam splitter (ABS) and steered toward the Wollaston prism (WP). To remove the problems related to the optical feedback and interference effects, ABS was set at an angle of about $10^{\circ}$ with respect to the output light wave front. By properly adjusting WP, the sampled light beam is split into two separate beams with linearly orthogonal polarizations associated with the principal axes of the laser. The signals related to the intensities of the two beams, detected by the photodetectors $\mathrm{PD}_{1}$ and $\mathrm{PD}_{2}$, are delivered to the stabilizing circuit.

The stabilizing circuit Figure 6 shows the stabilizing circuit in detail. At first, the switch $S$ is in position $A$, and the current of thermoelectric coolers, by changing the amplification coefficient $\left(R_{4}\right)$ and the input voltage $\left(R_{2}\right)$ of amplifier $\mathrm{OA}_{1}$, is adjusted so that the laser ambient 


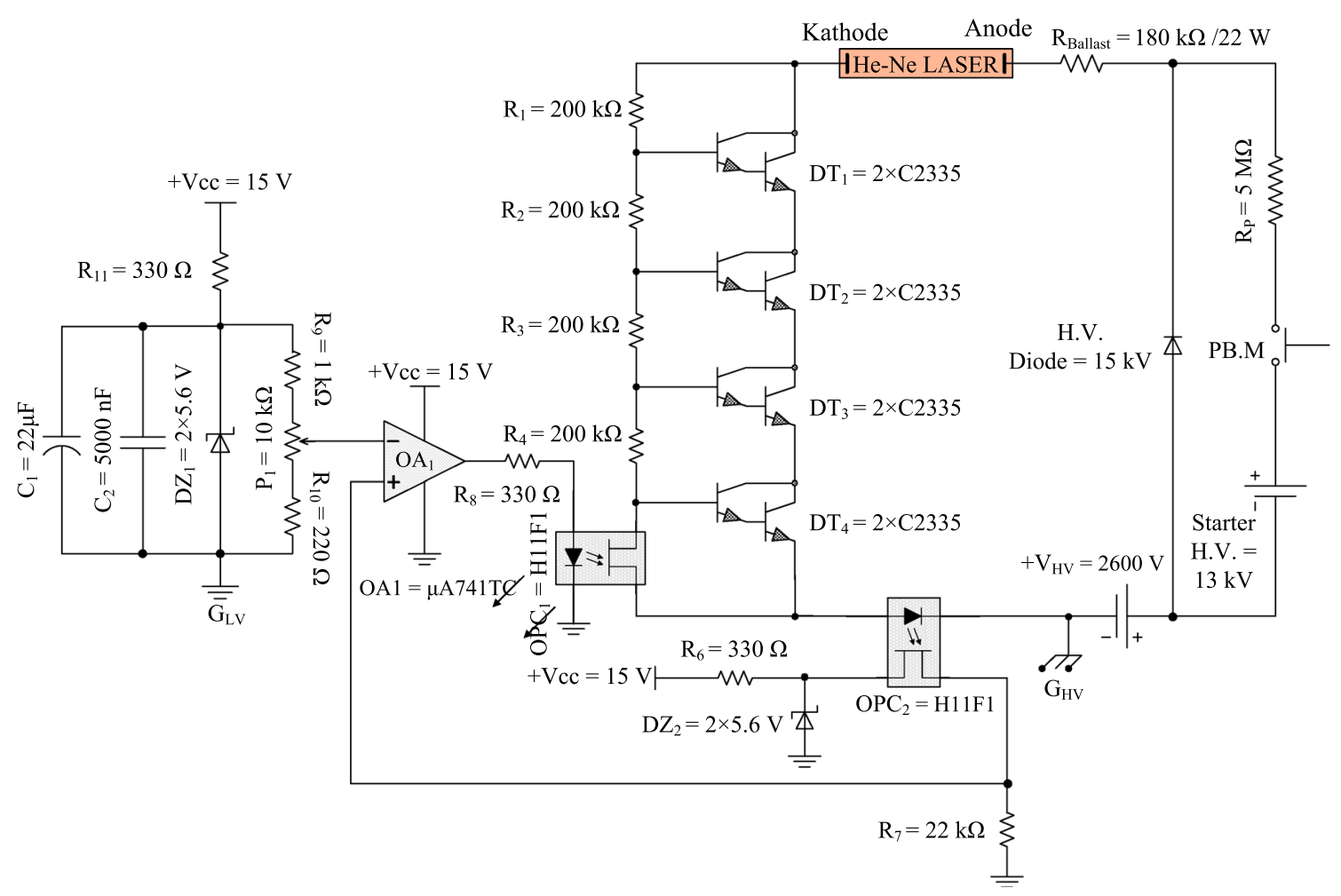

Fig. 4 The circuit of the power supply unit, including a high-voltage power supply and an adjustable current source

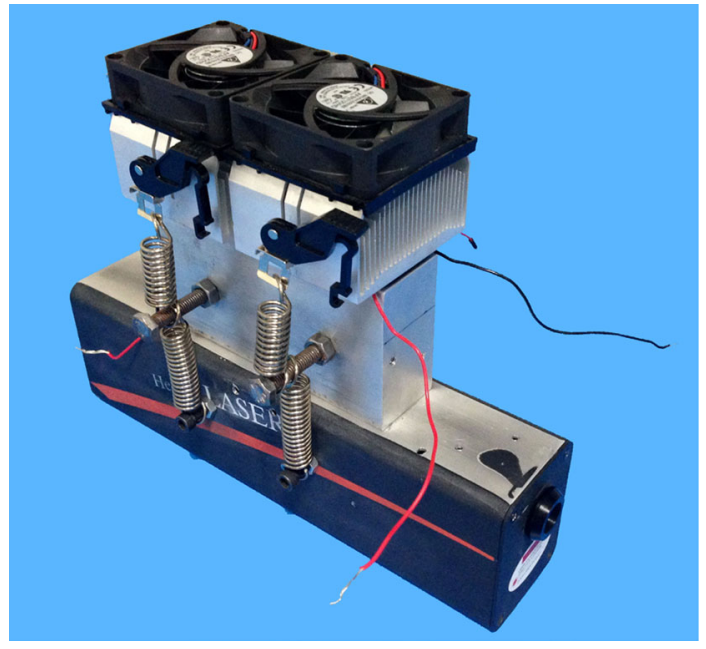

Fig. 5 The manner of connecting the two thermoelectric coolers on the laser box

temperature is fixed at the optimal temperature. The transistors $T_{1}, T_{2}$ and $T_{3}$ provide the necessary current for the thermoelectric coolers. $R_{17}$ and $R_{18}$ are ballast resistors that divide the coolers' current equally between $T_{2}$ and $T_{3}$. After fixing the temperature of the laser ambient, by putting the switch $S$ at position $B$, the control of the thermoelectric coolers is conceded to an integrator whose input is the signal of intensity difference of two principal modes.
The amounts of $R_{10}, C_{1}$ and also $R_{10} C_{1}$ are so determinant in the ultimate frequency stability, and normally they are empirically specified.

\section{Results and discussion}

Since in $\mathrm{He}-\mathrm{Ne}$ lasers, there exists an optimal current of laser tube in which the power of the laser light can be maximized [23], first of all, the laser power versus different discharge currents was measured by a power meter (including the optical sensor XLP12-3S-H2-D0 and the power monitor MAESTRO, from Gentec) at the equilibrium temperature of the laser with its ambient, $46^{\circ} \mathrm{C}$. Then, the best-fitting curve of the experimental points was plotted (Fig. 7). The figure shows that the optimal current of the laser tube corresponding to the maximum power is $5.5 \mathrm{~mA}$.

Therefore, to find the amount of ballast resistor $\left(R_{\text {Ballast }}\right)$ in Fig. 4, in addition to the current of the laser tube, the operating point voltage of the laser was also needed to be obtained. For this purpose, the voltage of the laser tube was measured versus different discharge currents using an adjustable current source. Figure 8 indicates the best characteristic curve of voltage versus the current of the laser tube (red line), plotted by the use of measured experimental data (blue dots). The operating point of the laser (Q) can be obtained from intersecting the DC load line of the laser 


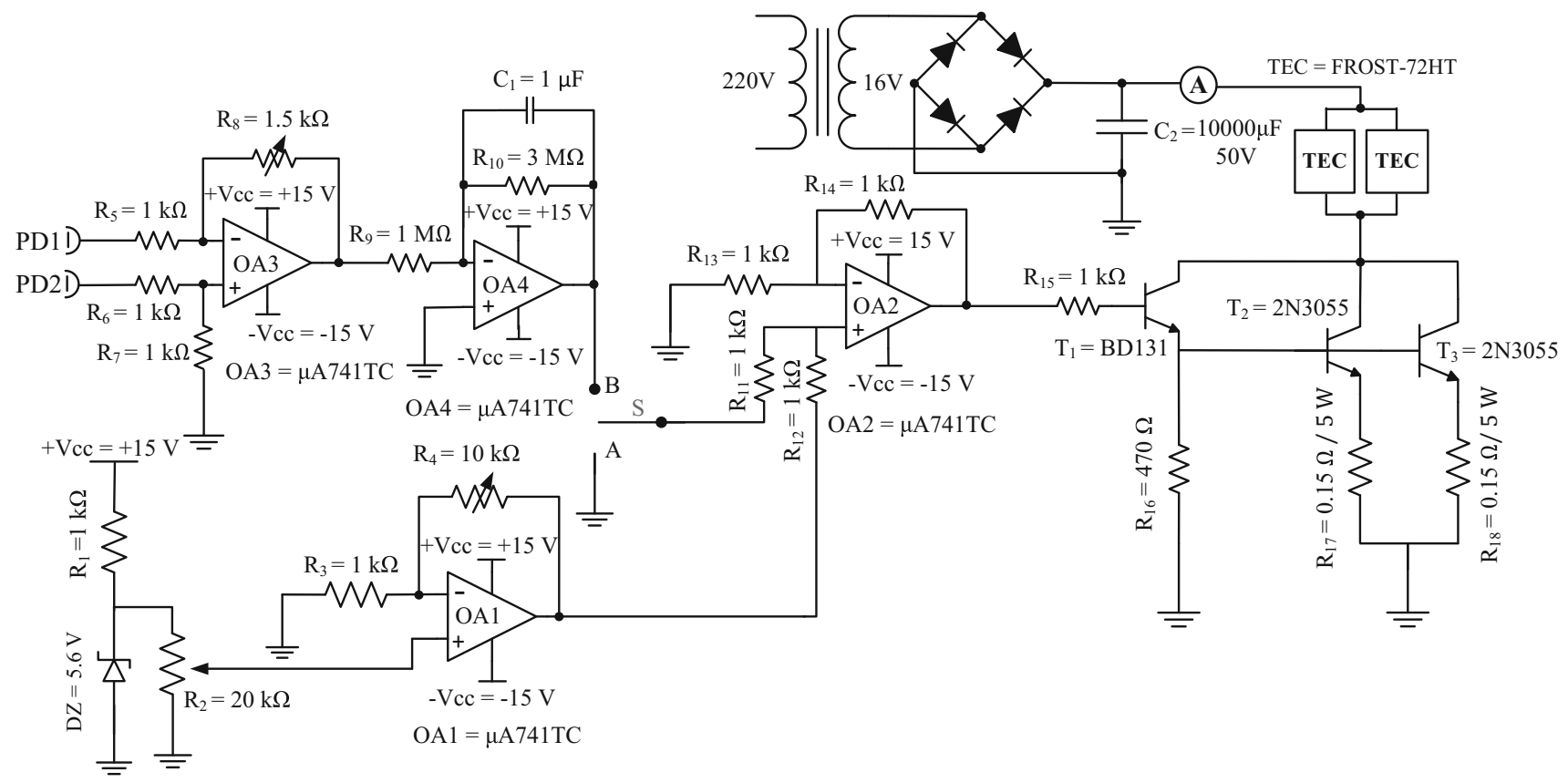

Fig. 6 The stabilizing system, including the feedback and cooling circuits

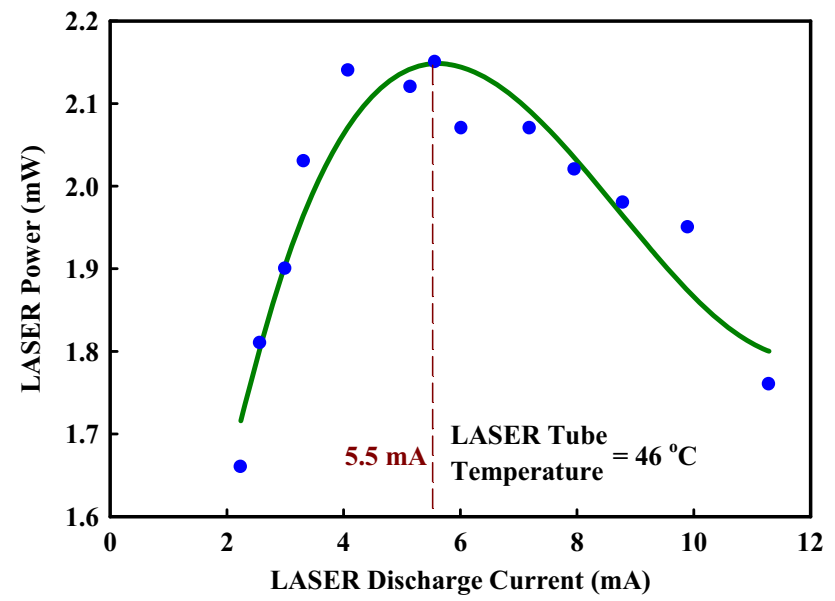

Fig. 7 The laser power versus discharge current: blue dots experimental data; green line the best-fitting curve of the experimental points

(green line), that is, $V_{\text {laser }}=2600-R_{B} I_{\text {laser }}(\mathrm{V})$, and the characteristic curve by considering $I_{\text {laser }}=I_{\text {opt }}=5.5 \mathrm{~mA}$.

The inspection indicated that the operating point voltage is equal to $1560 \mathrm{~V}$ and, therefore from the DC load line, $R_{B}$ was obtained to be $189.1 \mathrm{k} \Omega$. Since $R_{B}$ involves $R_{\text {Ballast }}$ and the equivalent resistor of the highvoltage transistors (Fig. 4), by considering the most stability of discharge performance of the laser, $R_{\text {Ballast }}$ was selected equal to $180 \mathrm{k} \Omega$. After adjusting the current of the laser tube to the optimal value, $5.50 \mathrm{~mA}$, it was a question for us if there was a temperature at which the laser output power was maximized.

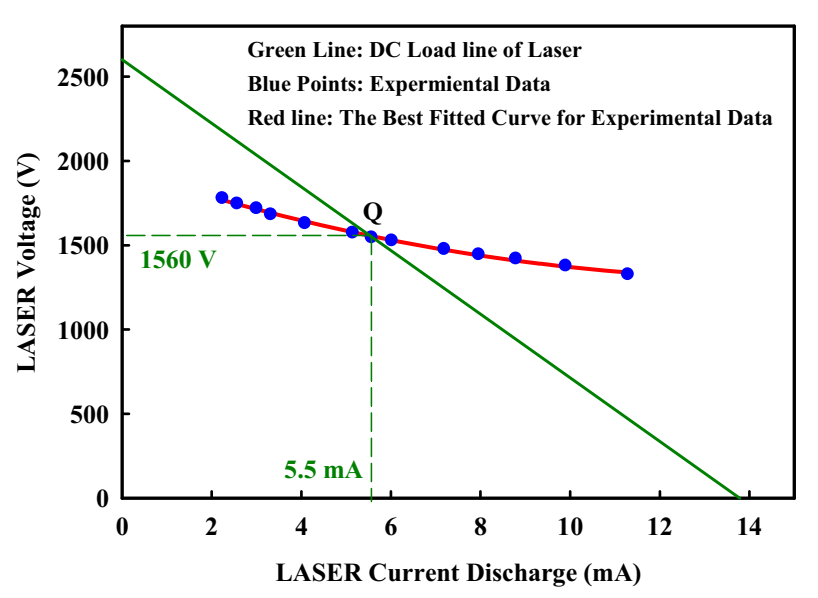

Fig. 8 The laser voltage versus discharge current, used for determining the operating point of the laser

To answer this, by using thermoelectric coolers, the laser output power in terms of different temperatures from 5 to $50{ }^{\circ} \mathrm{C}$ was measured and plotted (Fig. 9). As observed, the optimal temperature, that is, the temperature at which the laser power is the maximum, is about $23{ }^{\circ} \mathrm{C}$. Therefore, it is reasonable that the laser stabilization is carried out at the optimal temperature. The lasing at this temperature leads to two advantages: having maximum laser output power and increasing the laser tube lifetime owing to decreasing the temperature. Hence, by controlling the current of the Peltier thermoelectric coolers, the temperature of the laser was adjusted at $23{ }^{\circ} \mathrm{C}$. In this case, however, due to the variation of the laser tube temperature 


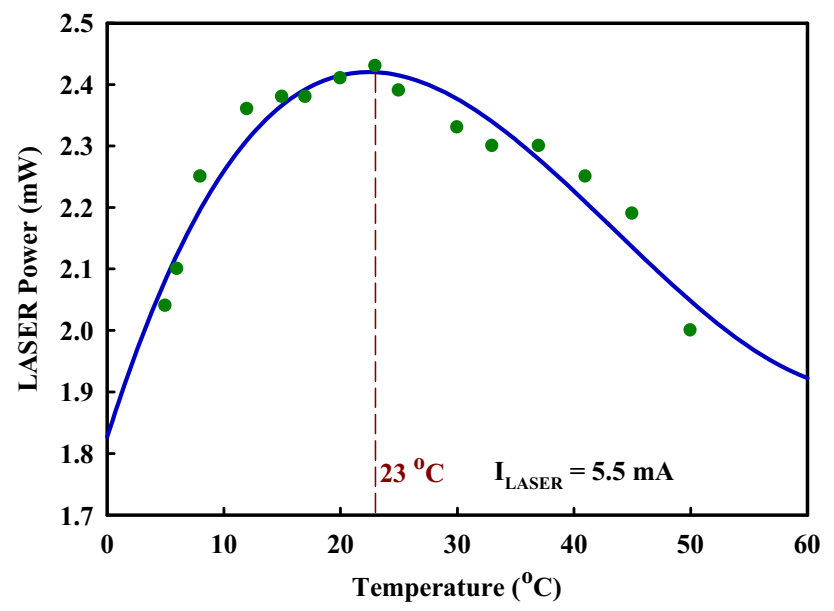

Fig. 9 The laser output power versus the tube temperature: green dots experimental data points; blue line the best-fitting curve of the experimental data

around the temperature $23{ }^{\circ} \mathrm{C}$, the length of the laser tube may alter, which in turn causes the movement of longitudinal modes on the gain profile of the laser and, consequently, an instability in the laser frequency. It is worth mentioning that before applying the stabilizing circuit to stabilize the frequency, the method of measuring the stability should be specified.

In order to measure the limit of stability, one can utilize the difference of two principal axes' intensities. Assume that $I_{x}$ and $I_{y}$ are the light intensities transmitted from the alignments of two principal axes indicated in Fig. 2. The temperature change results in the displacement of frequency modes on the line shape and, consequently, the variation of $\Delta I=I_{y}-I_{x}$. The zero points of $\Delta I$ correspond to the state in which the frequency of longitudinal modes stay on the same distance from the central frequency on the line shape (Fig. 10a).

The maxima and minima of $\Delta I$ occur when one of the two modes is at the central frequency. Therefore, the change of $\Delta I$ by $2 \Delta I_{\text {Max }}$ corresponds to the change of frequency of each longitudinal mode by the axial mode spacing $(\delta v=c / 2 L)$. Thus, one can write:

$\frac{\mathrm{d}(\Delta I)}{\mathrm{d} v}=\frac{2 \Delta I_{\mathrm{Max}}}{\delta v}$

This means that the stability $\left(\mathrm{d} v / v_{\text {osc }}\right)$ can be calculated by the following equation,

$\frac{\mathrm{d} v}{v_{\text {osc. }}}=\left(\frac{\mathrm{d}(\Delta I)}{2 \Delta I_{\mathrm{Max}}}\right) \frac{\delta v}{v_{\text {osc. }}}$.

By fixing the temperature at $23{ }^{\circ} \mathrm{C}$ and consequently the length of resonance cavity, it is expected that the frequency of the laser is stabilized on the gain profile; but the measurement of the laser modes via the method described in Fig. 1 showed that the frequency of each mode gradually

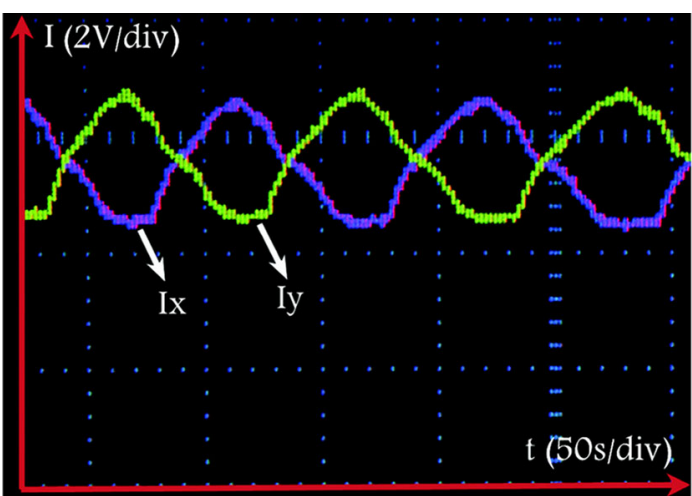

(a)

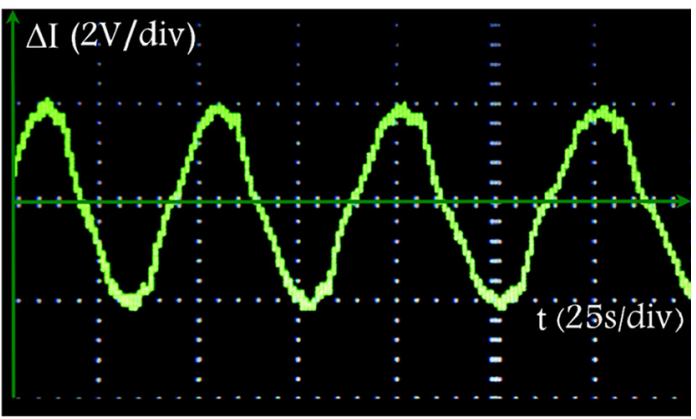

(b)

Fig. 10 Signals related to: a light intensities transmitted from the alignments of two principal axes, $I_{x}$ and $I_{y} ; \mathbf{b}$ the difference of intensities $\Delta I=I_{y}-I_{x}$

moves at a rate of $800 \mathrm{kHz} / \mathrm{s}$ on the gain lineshape, which is, according to Eq. (2), equivalent to short-term (1 min) stability of $4.30 \times 10^{-8}(\sim 70 \times)$. The displacement of laser oscillation modes on the line shape, by any reason, will be caused the change of $\Delta I$. Therefore, to overcome this variation, the switch $S$ (in Fig. 6) must be put at position $B$. Consequently, the feedback circuit including the integrator prevents the frequency variation by controlling the length of the resonance cavity toward compensating the changes. This process is carried out so that the laser frequency always stays constant on the gain profile. The measurement of stability using Eq. (2) indicates that the short- (less than $1 \mathrm{~min}$ ) and long- (more than $1 \mathrm{~h}$ ) term stabilities are equal to $1.17 \times 10^{-9}(\sim 2700 \times)$ and $2.57 \times 10^{-9}(\sim 1200 \times)$, respectively.

After having stabilized the laser frequency, the measurement through an SF-PI reflects that the laser operates in two longitudinal modes which are symmetrically spaced from the central frequency on the gain profile. This, of course, was expected, because in this case, as explained earlier, although $\Delta I=0$, the variation of $\Delta I$ is the maximum. On the other hand, since the input of the stabilizing circuit receives a signal corresponding to the variation of $\Delta I$, this means that the maximum sensitivity for detecting the fluctuation of frequency is associated with $\Delta I=0$, 
leading to a maximum frequency stabilization. Therefore, no matter in what state the control of the system to be transferred to the stabilizing circuit, the best stabilization is established at about $\Delta I=0$.

\section{Conclusion}

In this paper, an active method for frequency stabilization of an internal-mirror red He-Ne laser was scrutinized. First of all, the number of laser longitudinal modes and the direction of the laser principal axes were determined using a polarizer and an SF-PI. Then, the laser light power versus the discharge current was measured, and it was proved that there was an optimal current $(5.5 \mathrm{~mA})$ for which the laser power was the maximum. In addition, the measurement of the laser output power versus the temperature of the laser tube indicated that there was also an optimal temperature $\left(23{ }^{\circ} \mathrm{C}\right)$ in which the laser power was the maximum. Therefore, the current of the laser tube was set at $5.50 \pm 0.01 \mathrm{~mA}$ by an adjustable current source. Highprecision current source prevents any alteration or deviation from the optimal current due to power supply instability or city electricity's fluctuation, which, at first-order approximation, leads to a short-term stability of about $4.30 \times 10^{-8}$. Moreover, the laser system was isolated from the environment using two thermal and acoustic insulator shells. To appropriately control the temperature of the laser tube, two Peltier thermoelectric coolers were connected to the laser box through an aluminum block. By adjusting the current of the coolers, the laser tube was set at the optimal temperature. Then, to overcome any fluctuations in laser frequency, the laser tube's temperature was controlled by a feedback mechanism whose input receives a signal related to the difference of laser longitudinal modes' intensities. The measurement by the comparison method signified that the stability of laser frequency for less than $1 \mathrm{~min}$ (shortterm stability) is $1.17 \times 10^{-9}$, and for more than $1 \mathrm{~h}$ (longterm stability) is $2.57 \times 10^{-9}$, corresponding to a coherence length of the order $\sim 250 \mathrm{~m}$.

Open Access This article is distributed under the terms of the Creative Commons Attribution 4.0 International License (http://crea tivecommons.org/licenses/by/4.0/), which permits unrestricted use, distribution, and reproduction in any medium, provided you give appropriate credit to the original author(s) and the source, provide a link to the Creative Commons license, and indicate if changes were made.

\section{References}

1. Kano, K., Kozawa, Y., Sato, S.: Generation of a purely single transverse mode vortex beam from a He-Ne laser cavity with a spot-defect mirror. Int. J. Opt. 2012, 359141 (2012)
2. Vyas, S., Kozawa, Y., Sato, S.: Generation of a vector doughnut beam from internal mirror He-Ne laser. Opt. Lett. 39, 2080 (2014)

3. Balhorn, R., Kunzmann, H., Lebowsky, F.: Frequency stabilization of internal-mirror Helium-Neon lasers. Appl. Opt. 11, 742 (1972)

4. Bennett, S.J., Ward, R.E., Wilson, D.C.: Comments on frequency stabilization of internal mirror He-Ne lasers. Appl. Opt. 12, 1406 (1973)

5. Hamilton, M.W.: An introduction to stabilized lasers. Contem. Phys. 30, 21 (1989)

6. Yoshino, T.: Frequency stabilization of internal-mirror $\mathrm{He}-\mathrm{Ne}$ $(\lambda=633 \mathrm{~nm})$ lasers using polarization properties. Jpn. J. Appl. Phys. 19, 2181 (1980)

7. Yokoyama, Sh, Araki, T., Oshio, T., Suzuki, N.: Frequency stabilization of a multimode high-power He-Ne laser. Rev. Sci. Instrum. 64, 2796 (1993)

8. Budzyn, G., Rzepka, J.: Back-reflection effects in a frequencystabilized two-mode He-Ne laser. Opt. Commun. 281, 5592 (2008)

9. Eom, T.B., Choi, H.S., Lee, S.K.: Frequency stabilization of an internal mirror He-Ne laser by digital control. Rev. Sci. Instrum. 73, 221 (2002)

10. Diao, X., Tan, J., Hu, P., Yang, H., Wang, P.: Frequency stabilization of an internal mirror He-Ne laser with a high frequency reproducibility. Appl. Opt. 52, 456 (2013)

11. Araki, T., Nakajima, Yu., Suzuki, N.: Frequency and intensity stabilization of a high output power, internal mirror He-Ne laser using interferometry. Appl. Opt. 28, 1525 (1989)

12. Mio, N., Ko, M.-F., Ni, W.-T., Pan, S., Araya, A., Moriwaki, S., Tsubono, K.: Design of a stabilized He-Ne laser by using a thinfilm heater. Appl. Opt. 32, 5944 (1993)

13. Umeda, N., Tsukiji, M., Takasaki, H.: Stabilized 3 He-20 Ne transverse Zeeman laser. Appl. Opt. 19, 442 (1980)

14. Sasaki, A., Hayashi, T.: Amplitude and frequency stabilization of an internal-mirror He-Ne laser. Jpn. J. Appl. Phys. 21, 1455 (1982)

15. Qian, J., Liu, Z., Shi, C., Liu, X., Wang, J., Yin, C., Cai, S.: Frequency stabilization of internal-mirror $\mathrm{He}-\mathrm{Ne}$ lasers by air cooling. Appl. Opt. 51, 6084 (2012)

16. Ellis, J.D., Joo, K.-N., Buice, E.S., Spronck, J.W.: Frequency stabilized three mode HeNe laser using nonlinear optical phenomena. Opt. Express 18, 1373 (2010)

17. Ellis, J.D., Voigt, D., Spronck, J.W., Verlaan, A.L., Munnig Schmidt, R.H.: Frequency stabilized HeNe gas laser with $3.5 \mathrm{~mW}$ from a single mode. Precis. Eng. 36, 203 (2012)

18. Persson, W., Svanberg, J.: Laser spectroscopy VIII. Springer, Berlin (1987)

19. Sasaki, Akira, Ushimaru, Shinji, Hayashi, Takao: Simultaneous output-and frequency-stabilization and single-frequency operation of an internal-mirror $\mathrm{He}-\mathrm{Ne}$ laser by controlling the discharge current. Jpn. J. Appl. Phys. 23, 593 (1984)

20. Winkler, W., Rudger, A., Schilling, R., Strain, K.A., Danzmann, K.: Birefringence-induced losses in interferometers. Opt. Commun. 112, 245 (1994)

21. Yoshino, T.: Polarization properties of internal-mirror $\mathrm{He}-\mathrm{Ne}$ lasers at 6328 A. Jpn. J. Appl. Phys. 11, 263 (1972)

22. Yoshino, T.: Reflection anisotropy of 6328 A laser mirrors. Jpn. J. Appl. Phys. 18, 1503 (1979)

23. Döhner, H.-J., Elsässer, W.: Analysis of a four-level laser system: investigations of the output power characteristics of a $\mathrm{He}-\mathrm{Ne}$ laser. Am. J. Phys. 59, 327 (1991) 